\title{
Henoch-Schönlein purpura in an elderly patient with unusual manifestation
}

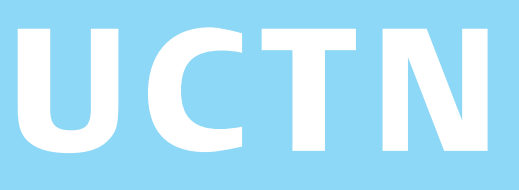

An 81-year-old man was admitted to the hospital with hematochezia and ascites, but with no abdominal pain or skin rash. Colonoscopy revealed a circumferential ulcer with marked edema in the terminal ileum (Figure 1a). Duodenoscopy also showed multiple ulcers in the duodenum (Figure $\mathbf{1} \mathbf{b}$ ). Histological examination of biopsy specimens indicated nonspecific inflammation. Paracentesis yielded hemorrhagic fluid, with no malignant cells and no growth of pathogens. A definite diagnosis could not be established initially, and the patient was treated conservatively.

On hospital day 8, palpable purpuric lesions abruptly developed at both extremities, hematochezia progressed, and renal function rapidly worsened. On the basis of these findings, Henoch-Schönlein purpura was diagnosed clinically, and was pathologically confirmed several days later (Figure 2). Despite intensive treatment, including methylprednisolone pulse therapy, the patient's condition progressively deteriorated and he died on day 13.

Autopsy revealed massive hemorrhagic ascites and coagulated blood in the gastrointestinal tract. The mucosa in both the duodenum and the ileum was extensively peeling, but interestingly, the jejunal and colonic mucosa was intact (Figure 3). Immunohistochemistry demonstrated IgA depositions on the small vessels of the bowel wall, including not only the duodenum and the ileum, with extensive ulcers, but also the jejunum, with a normal appearance.

The presence of characteristic purpura can facilitate the diagnosis of HenochSchönlein purpura, but the diagnosis is more difficult when other manifestations of the disease precede the purpura [1-4]. In the present patient, it was difficult to obtain a final diagnosis of Henoch-Schönlein purpura, as the case had the following unusual in characteristics: old age at onset, delayed appearance of characteristic

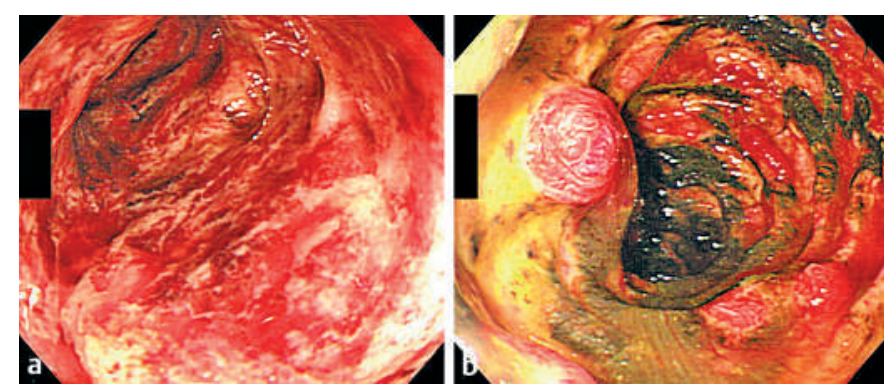

Figure 1 Endoscopic views. a Colonoscopy, showing a circumferential ulcer with marked edema in the terminal ileum. b Duodenoscopy, showing multiple ulcers in the second part of the duodenum.

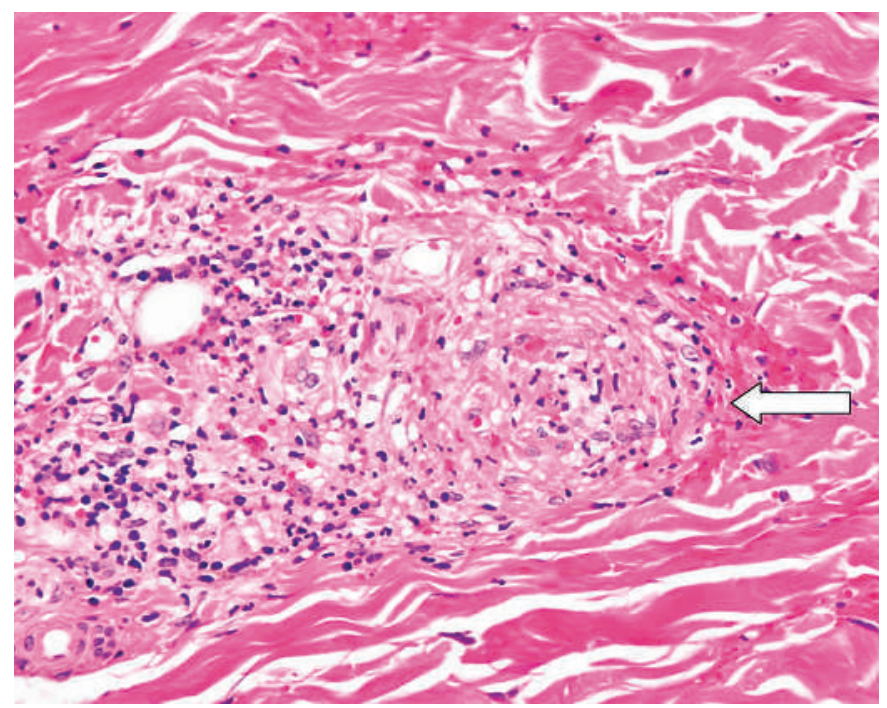

Figure 2 A microscopic view of the skin. Hematoxylineosin stain, showing leukocytoclastic vasculitis (arrow), which is representative of Henoch-Schönlein purpura.
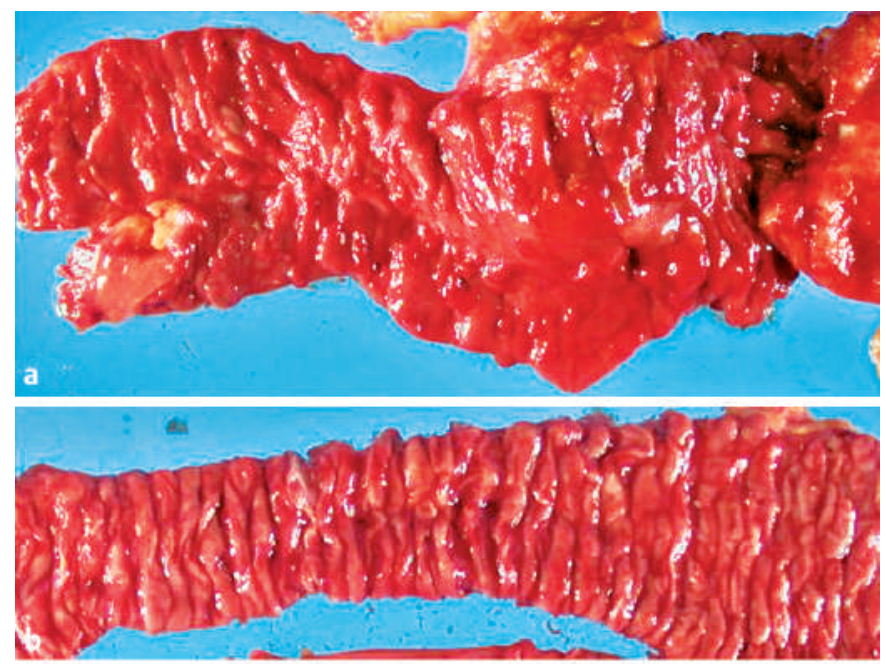

Figure 3 Macroscopic views of the gastrointestinal tract at autopsy. a Multiple ulcers were observed in the duodenum. $\mathbf{b}$ The jejunal mucosa was intact. c The mucosa in the ileum was extensively peeling, with pseudopolyps.

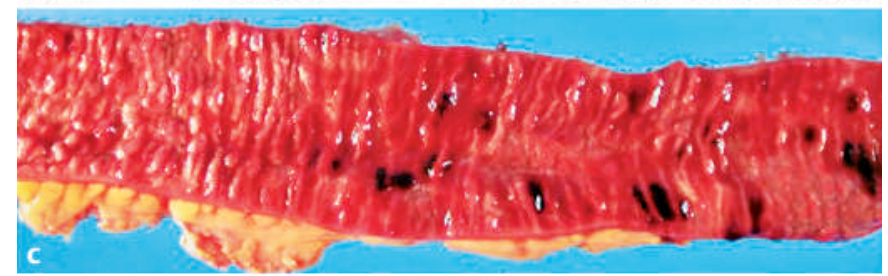

DOI: 10.1055/s-2006-945022 


\section{UCTN}

purpura, an absence of abdominal pain, and the presence of hemorrhagic ascites. The patient also had an unusual distribution of gastrointestinal lesions, with the jejunum being spared despite systemic vasculitis. However, the reason for the absence of the jejunal lesions is not clear.

Endoscopy_UCTN_Code_CCL_1AD_2AF

Endoscopy_UCTN_Code_CCL_1AB_2AZ_3AD

N. Uza' ${ }^{1}$, S. Yazumi'1, K. Tanabe', Y. Endo', Y. Kodama', Y. Matsumura ${ }^{2}$,

H. Sakashita ${ }^{3}$, T. Chiba ${ }^{1}$

${ }^{1}$ Dept. of Gastroenterology and Hepatology

2 Dept. of Dermatology

${ }^{3}$ Dept. of Pathology, Graduate School of Medicine, Kyoto University, Kyoto, Japan.
References

${ }^{1}$ Saulsbury FT. Henoch-Schönlein purpura. Curr Opin Rheumatol 2001; 13: 35-40

2 Godkin A, Thompson M, Summerfield J. Abdominal pain and melaena: an unusual cause. Lancet 2000; 356: $562-563$

${ }^{3}$ Kim HS, Lee DK, Baik SK et al. Ileal vasculitis in Henoch-Schönlein purpura. Gastrointest Endosc 2001; 54: 493-494

${ }^{4}$ Sasaki K, Nukuda Y, Masuda T et al. Endoscopically and histologically documented gastrointestinal lesions in an adult patient with Henoch-Schönlein purpura. Endoscopy 1994; 26: 629-630
Corresponding author

S. Yazumi, M.D., Ph.D.

Dept. of Gastroenterology and Hepatology Kyoto University

Graduate School of Medicine

Shogoin-Kawara-cho, Sakyo-ku

Kyoto 606-8507

Japan

Fax: $\quad+81-75-751-4303$

Email: yazoo@kuhp.kyoto-u.ac.jp 